PAPER

Deposition in the inner and outer corners of the JET divertor with carbon wall and metallic ITERlike wall

To cite this article: J Beal et al 2016 Phys. Scr. 2016014052

View the article online for updates and enhancements.
Related content - Overview of fuel inventory in JET with the
$\frac{\text { ITER-like wall }}{\text { A. Widdowson, J.P. Coad, E. Alves et al. }}$
- Time-resolved deposition in the remote
$\frac{\text { region of the JET-ILW divertor: }}{\text { measurements and modelling }}$
N Catarino, A Widdowson, A Baron-
Wiechec et al.
- Erosion and deposition in the JET divertor
during the second ITER-like wall campaign
M Mayer, S Krat, A Baron-Wiechec et al.

Recent citations

- Comparison of erosion and deposition in JET divertor during the first three ITER-like wall campaigns S Krat et al

Erosion at the inner wall of JET during the discharge campaign 2013-2014

S. Krat et al

Overview of the JET ITER-like wall divertor A. Widdowson et al 


\title{
Deposition in the inner and outer corners of the JET divertor with carbon wall and metallic ITER-like wall
}

\author{
J Beal $^{1,2}$, A Widdowson ${ }^{2}$, K Heinola ${ }^{2,3}$, A Baron-Wiechec ${ }^{2}, \mathbf{K}$ J Gibson ${ }^{1}$, \\ J P Coad ${ }^{2}$, E Alves ${ }^{4}$, B Lipschultz ${ }^{1}$, A Kirschner ${ }^{5}$, H G Esser ${ }^{5}$, \\ G F Matthews ${ }^{2}$, S Brezinsek ${ }^{5}$ and JET Contributors ${ }^{6}$ \\ EUROfusion Consortium, JET, Culham Science Centre, Abingdon, OX14 3DB, UK \\ ${ }^{1}$ Department of Physics, York Plasma Institute, University of York, Heslington, York, YO10 5DD, UK \\ ${ }^{2}$ Culham Centre for Fusion Energy, Abingdon, OX14 3DB, UK \\ ${ }^{3}$ University of Helsinki, PO Box 64, FI-00014, University of Helsinki, Finland \\ ${ }^{4}$ Instituto de Plasmas e Fusão Nuclear, Instituto Superior Técnico, Universidade de Lisboa, Avenue \\ Rovisco Pais, 1049-001, Lisboa, Portugal \\ ${ }^{5}$ Forschungszentrum Jülich, Institut für Energie- und Klimaforschung Plasmaphysik, D-52425 Jülich, \\ Germany
}

E-mail: James.Beal@ccfe.ac.uk

Received 19 May 2015

Accepted for publication 30 June 2015

Published 21 January 2016

\begin{abstract}
Rotating collectors and quartz microbalances (QMBs) are used in JET to provide time-dependent measurements of erosion and deposition. Rotation of collector discs behind apertures allows recording of the long term evolution of deposition. QMBs measure mass change via the frequency deviations of vibrating quartz crystals. These diagnostics are used to investigate erosion/deposition during JET-C carbon operation and JET-ILW (ITER-like wall) beryllium/ tungsten operation. A simple geometrical model utilising experimental data is used to model the time-dependent collector deposition profiles, demonstrating good qualitative agreement with experimental results. Overall, the JET-ILW collector deposition is reduced by an order of magnitude relative to JET-C, with beryllium replacing carbon as the dominant deposit. However, contrary to JET-C, in JET-ILW there is more deposition on the outer collector than the inner. This reversal of deposition asymmetry is investigated using an analysis of QMB data and is attributed to the different chemical properties of carbon and beryllium.
\end{abstract}

Keywords: tokamak, JET, erosion, deposition

(Some figures may appear in colour only in the online journal)

\section{Introduction}

Erosion, deposition and material migration can limit the lifetime of fusion tokamaks and degrade plasma performance. The material composition of plasma-facing surfaces strongly affects the scale and nature of plasma-material interaction processes. The carbon wall of JET-C has been replaced with the beryllium main chamber and tungsten divertor of JET-

\footnotetext{
6 See the appendix of F Romanelli et al Proc. 25th IAEA Fusion Energy Conference 2014, Saint Petersburg, Russia.
}

ILW (ITER-like wall) [1], leading to beryllium replacing carbon as the dominant plasma impurity [2]. The understanding of erosion/deposition with this new material combination provides vital experience for efficient operation of ITER. The accumulation of deposits in remote tokamak areas can contribute significantly to fuel retention [3]. This study examines erosion/deposition in the inner and outer corners of the JET-C and JET-ILW divertors. Post-mortem tile analysis offers the most comprehensive test of changes to plasmafacing surfaces, but only reveals the aggregated effects of a 1-2 year operating campaign. Rotating collectors and quartz 


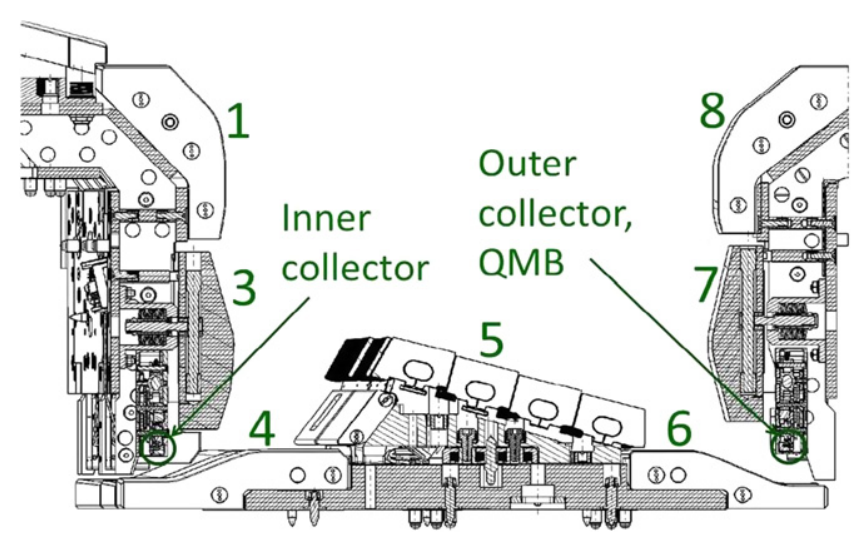

Figure 1. Poloidal cross-section of the JET divertor, showing tile numbers and locations of relevant rotating collectors and QMBs.

microbalances (QMBs) offer improved time resolutions, allowing deposition to be related to varying plasma conditions.

\section{Experimental}

Rotating collectors consist of silicon discs that act as substrates for deposition and are driven to rotate incrementally by pulsing of the toroidal magnetic field [4]. Each disc is housed behind an aperture that exposes a thin sector of the disc to the plasma. Gradual rotation of the disc yields a $\sim 60$ discharge moving sum of deposition around its face. The carbon, beryllium and deuterium deposits are simultaneously quantified ex situ by nuclear reaction analysis and mapped to discharge number, allowing the effects of different plasma configurations/parameters to be investigated.

QMBs exploit the mass dependence of the frequency of a vibrating quartz crystal to determine deposition on or erosion from the crystal [5]. The crystals are housed behind shutters that are opened for several seconds during a discharge, allowing the erosion/deposition during this period to be inferred from the frequency change. Since the frequency is also dependent on the crystal temperature, the frequency change of a second, unexposed crystal is subtracted in order to isolate the effects of erosion/deposition alone.

Rotating collectors in the inner and outer corners of the JET divertor (figure 1) are analysed for 2005-07 carbon and 2010-12 ILW operation. Unfortunately, failure of the invessel QMB electronics in 2010-12 means that 2005-07 and 2013-14 QMB data is used to examine outer divertor erosion/deposition. Although there were differences in operating conditions between 2010-12 and 2013-14, a general analysis of the effects of wall materials and strike point locations is unlikely to be affected significantly.

\section{Modelling}

The time-dependent collector deposition is modelled using a simple geometrical approach employing a range of experimental data [6]. Since thousands of discharges are modelled, the description of transport is necessarily simplified using line of sight assumptions. Impurities are launched from time-varying strike point locations in cosine angular distributions and integrated over the acceptance angles of the collectors. A moving integral is applied to the modelled collector depositions to reproduce the effect of the finite aperture width. The focus is on variations in deposition, with modelled deposition profiles scaled by (constant, $<1$ ) attenuation factors. These are found by fitting to experimental deposition magnitudes and describe the proportion of eroded neutrals that remain un-ionised by the plasma, thus being able to reach the collector surfaces.

The $\mathrm{C}$ and Be erosion yields are calculated analytically using [7, 8]. The carbon case includes the chemical sputtering/erosion of amorphous hydrocarbon layers. Incident deuterium energies were estimated as $E_{\mathrm{i}}=3 Z T_{\mathrm{e}}+2 T_{\mathrm{i}} \approx 5 T_{\mathrm{e}}$, with electron temperature $T_{\mathrm{e}}$, ion temperature $T_{\mathrm{i}}=T_{\mathrm{e}}$, and atomic number $Z$. Assuming $T_{\mathrm{e}}=10 \mathrm{eV}, 25 \mathrm{eV}$ gives $E_{\mathrm{i}}=50 \mathrm{eV}, 125 \mathrm{eV}$ at the inner/outer strike points respectively. Incident ion fluxes were obtained from local Langmuir probes, or scaled from energies deposited at tiles via a power balance when probe data were unavailable. The fluxes are multiplied by yields to calculate the sputtering sources. The flux and the tile temperature (found from IR thermography/ thermocouples) also affect the calculated carbon chemical erosion yield [7].

The reduced impurity concentration in JET-ILW [9] leaves less Be available for sputtering in the divertor. Be ions in the incident flux that impact a bare tungsten divertor tile are reflected efficiently as neutral atoms [10]. Thus, deposition on the collectors may occur via either sputtering or reflection from tiles, depending on the local Be surface coverage. In order to incorporate the effects of varying levels of $\mathrm{Be}$ in the incident flux and on the tile surfaces, the sources were scaled by spectroscopic Be signal magnitudes using views of tile surfaces coinciding with strike points.

\section{Results}

The experimental/modelled deposition profiles on the inner and outer collectors show good qualitative agreement (figures 2 and 3). Initial attempts to model the JET-C outer divertor deposition (figure 3(a)) yielded poor agreement with experiment in likeness and magnitude. The reasonable agreement seen is the result of assuming that only tile 5 strike points caused deposition on the JET-C collector. Superficially this seems flawed due to the proximity of the collector to tile 6 (see figure 1). However, this was prompted by an analysis of QMB data, which indicates that the simplified geometrical description is not necessarily appropriate for JET-C tile 6 strike points (see section 5).

The replacement of carbon impurities with beryllium [2] was replicated in the collector results. For JET-C, the carbon and deuterium deposition profiles showed a strong similarity, indicating codeposition. For JET-ILW, this similarity of C and $\mathrm{D}$ profiles was only preserved in the inner divertor at a 

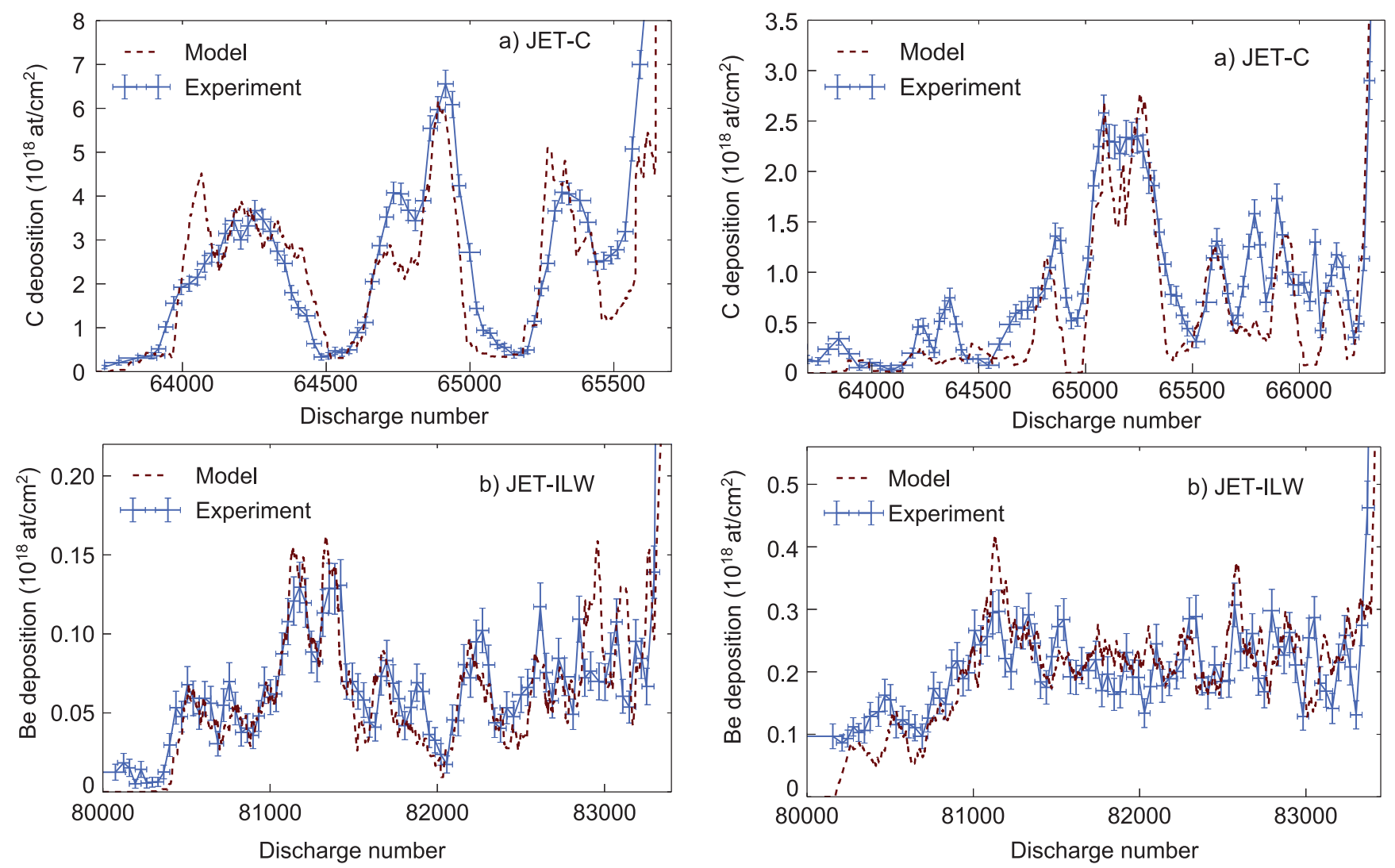

Figure 2. Experimental and modelled (multiplied by attenuation factors) inner divertor collector deposition: (a) JET-C (attenuation factor 0.76), (b) JET-ILW (attenuation factor 0.88).

reduced magnitude of $10-20 \%$ of the Be deposition. Rutherford Backscattering Spectrometry was used to identify total tungsten deposits of order $10^{16}$ at $\mathrm{cm}^{-2}$ aggregated over the campaign. The higher plasma temperatures in the outer divertor should result in greater tungsten erosion, but they also decrease the (already short) tungsten ionisation lengths, promoting rapid local redeposition.

While the collectors yield time-varying deposition data, the averaged deposition rates also offer important insights (figure 4). Overall, the deposition rate on the JET-ILW divertor corner collectors is an order of magnitude lower than in JET-C. In JET-C, $\sim 75 \%$ of the total deposition was on the inner collector, whereas in JET-ILW the proportions are reversed and the outer collector dominates deposition.

The cumulative frequency evolutions of outer divertor QMBs are shown in figure 5. The measurements for JET-C and JET-ILW have each been divided into two datasets depending on whether the strike point was on tile 5 or 6 , allowing the effects of the different configurations to be identified. The JET-C QMB generally shows net deposition when the strike point is on tile 5 and net erosion when it is on tile 6. Conversely, the JET-ILW collector shows net deposition for both tile 5 and tile 6 strike points.

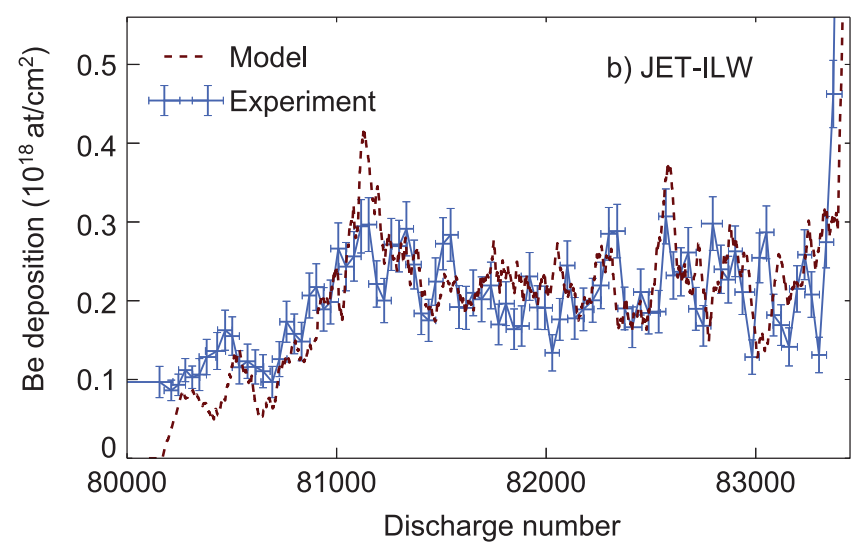

Figure 3. Experimental and modelled (multiplied by attenuation factors) outer divertor collector deposition: (a) JET-C (attenuation factor 0.26), (b) JET-ILW (attenuation factor 0.17).

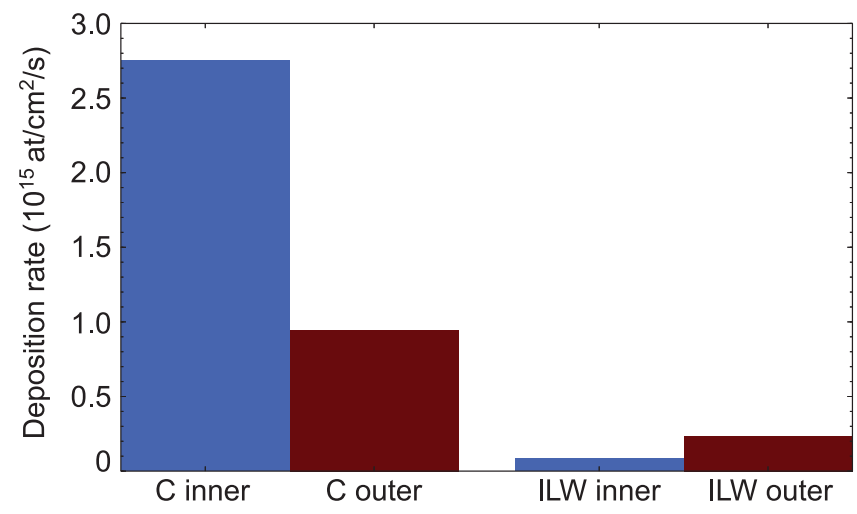

Figure 4. The large reduction in deposition between JET-C and JETILW collectors is asymmetric. In JET-C, the inner divertor dominates deposition, while in JET-ILW the outer dominates.

\section{Discussion}

The order of magnitude reduction in deposition for JET-ILW is consistent with results from rotating collectors under tile 5 [6], and with overall divertor deposition [11]. The primary cause is the reduction in the beryllium main chamber source relative to the JET-C carbon source, causing a factor 7 decrease in impurity migration to the divertor [12]. Additionally, unlike carbon, beryllium is not subject to thermally- 

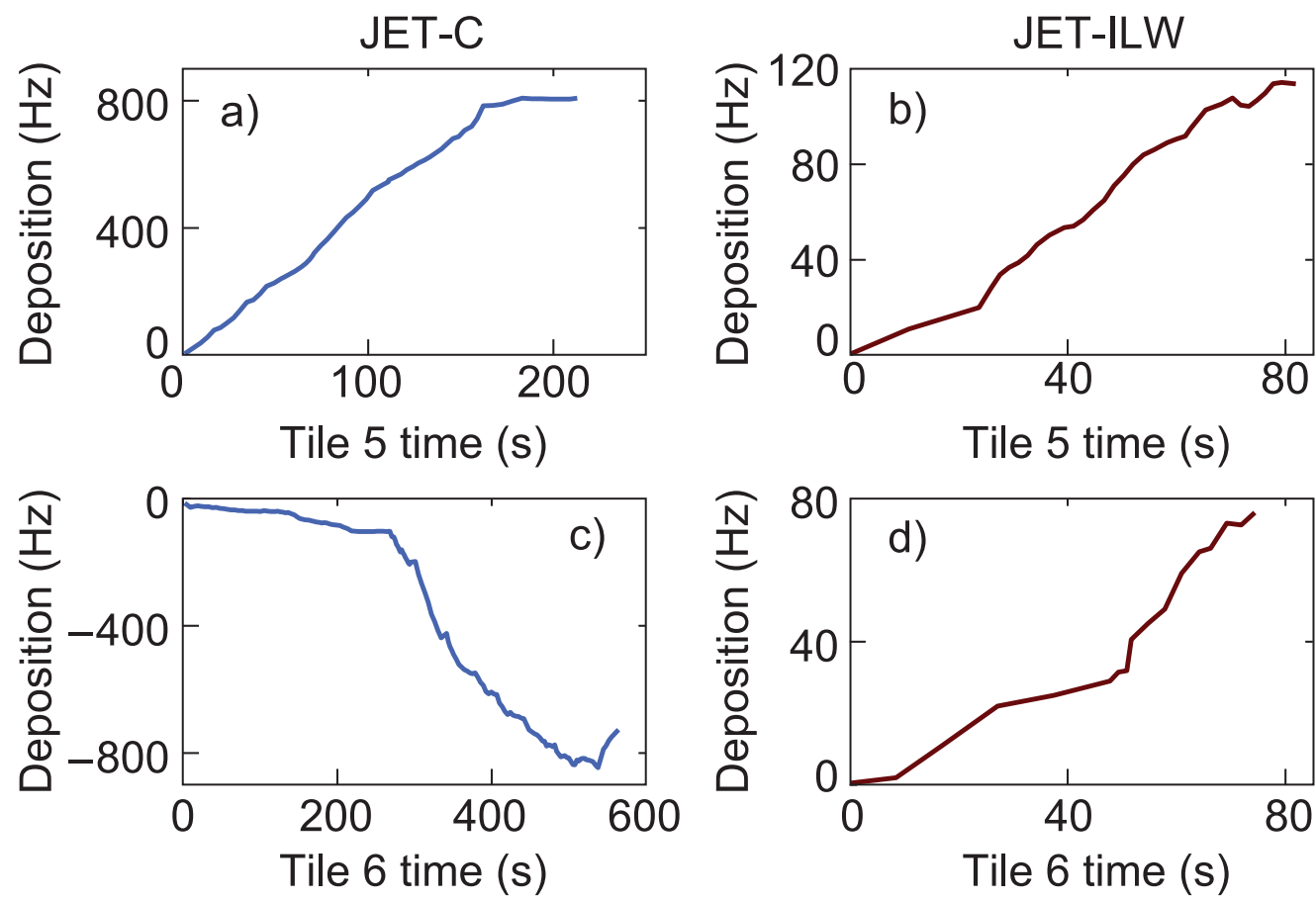

Figure 5. Cumulative changes in outer divertor QMB frequencies while strike points were on tile 5 or tile 6. (a) tile 5, JET-C (b) tile 5, JETILW, (c) tile 6, JET-C, (d) tile 6, JET-ILW. Increasing frequencies indicate deposition on QMBs; decreasing frequencies indicate erosion.

activated chemical erosion, with physical sputtering (and some chemically assisted physical sputtering) dominating [13]. Finally, the proportion of tile 4 strike points (close to the inner collector) in JET-ILW was reduced by a factor of 2.1 .

Greater deposition has generally been observed in the JET-C inner divertor than the outer, due to outboard to inboard SOL flows [14] and the dependence of impurity sticking on surface temperature [15]. This is reflected in the larger deposition rate seen on the inner JET-C collector than the outer (figure 4). When the outer strike point is on tile 6 , the JET-C QMB shows net erosion (figure 5(c)). These strike points cause high surface temperatures in the outer corner, as observed from local thermocouples. For the relatively low temperatures in the shadowed divertor corners, the chemical re-erosion increases with substrate temperature [7]. Thus, the net erosion is attributed to high QMB temperatures limiting deposition and promoting chemical/thermal re-erosion of carbon deposits. Tile 5 strike points result in lower outer corner surface temperatures, allowing net deposition on the QMB (figure 5(a)).

The QMB and collector have a narrow line of sight to tile 5 , but a contribution to the deposition is also probable from the tail of the strike point, close to the outer corner. During ELMs, it is possible for a fraction of very energetic ions to undergo multiple erosion/reflection steps, contributing an additional non-line of sight erosion source. Inner divertor strike points may also contribute to JET-C outer divertor deposition [16]. The outer divertor QMB has a partial view of tiles 1 and 3 , but not of tile 4 . Tile $1 / 3$ inner strike points generally pair with tile 5 outer strike points, and tile 4 with tile 6. This offers an additional explanation for the configuration dependence of the QMB erosion/deposition.
The modelling of the deposition on the JET-C outer divertor collector assumed that deposition occurred only for tile 5 strike points, but figure 5(c) suggests that re-erosion for tile 6 strike points should also be applied. In practice, the collector surface temperature is likely to be significantly lower than that of the QMB (and more representative of wall temperatures). In [4], the greater thermal isolation of a QMB is inferred to have lowered the deposition by a factor 4 relative to a co-located collector.

Without direct surface temperature measurements, it was decided inappropriate to extrapolate erosion rates found from the QMB to the collector. The JET-C outer collector temperature can at least be said to be higher than that of the inner collector, as shown by the lower deuterium to carbon ratio of $\sim 0.5$, compared to $\sim 1$ in the inner divertor. The blunt assumption of no net erosion or deposition for tile 6 strike points is preferred to more complex and uncertain conjectures. This modelling approach, though it does not yield as strong a match with experiment as for the other collectors, nevertheless shows reasonable agreement (figure 3(a)). For example, the period of discharges from $\sim 65000$ to 65350 had consistent strike points on tile 5 , resulting in a strong peak in collector deposition. The attenuation factor of 0.26 neglects tile 6 strike points and is thus not directly comparable to the other values. In terms of matching magnitudes, including all strike points would require reducing this factor to 0.03 , suggesting significant ionisation/redeposition/re-erosion for tile 6 strike points. The attenuation factors are currently being investigated using Monte Carlo modelling of impurity divertor transport.

The JET-ILW outer QMB (figures 5(b) and (d)) shows net deposition for both tile 5 and tile 6 strike points, suggesting the high outer divertor surface temperatures do not affect the 
erosion/deposition balance in the same way. Beryllium deposits on the JET-ILW QMB will not suffer thermallyinduced chemical erosion [13], resulting in consistent net deposition. The contribution of both configurations to deposition on the JET-ILW outer divertor collector is seen firstly in the fairly constant deposition profile (figure 3(b)), and secondly in the relatively high averaged deposition rate (figure 4). This leads to a reversal in deposition asymmetry in JET-ILW, with more deposition seen on the outer collector than the inner. Larger beryllium deposits in the outer divertor than the inner have also been found on louvre clips in the remote divertor corners [17], test mirrors in similar locations to the collectors [18], and the horizontal base tiles 4 and 6 [19].

\section{Conclusions}

Rotating collectors, QMBs and a geometrical modelling approach have been used to investigate erosion/deposition in the JET divertor corners. The qualitative agreement of collector results and modelled deposition profiles indicates the continued importance of line of sight transport of neutral impurities from divertor tiles to remote regions. Overall, the JET-ILW collectors showed an order of magnitude less deposition than the JET-C collectors. This was caused by a lower main chamber source, reduced chemical sputtering/ erosion and changes in the (inner divertor) strike point distribution. In JET-C, the inner divertor collector dominated the total deposition, but in JET-ILW most deposition was seen on the outer collector. This reversal of deposition asymmetry has been investigated using QMBs, and is contributed to by the decreased dependence of beryllium erosion/deposition on surface temperature via chemical effects. Further modelling is required to investigate any differences in global impurity transport processes between the JET-C and JET-ILW divertors in light of the deposition results.

\section{Acknowledgements}

This work has been carried out within the framework of the EUROfusion Consortium and has received funding from the Euratom research and training programme 2014-2018 under grant agreement No 633053. The views and opinions expressed herein do not necessarily reflect those of the European Commission.

\section{References}

[1] Matthews G F et al 2011 Phys. Scr. T 145014001

[2] Brezinsek S et al 2013 J. Nucl. Mater. 438 S303

[3] Federici G et al 1999 J. Nucl. Mater. 266-9 14

[4] Coad J P et al 2009 Phys. Scr. T 138014023

[5] Esser H G et al 2003 Fusion Eng. Des. 66-8 855

[6] Beal J et al 2015 J. Nucl. Mater. 463818

[7] Roth J and Garcia-Rosales C 1996 Nucl. Fusion 361647

[8] Eckstein W and Preuss R 2003 J. Nucl. Mater. 320209

[9] Coenen J W et al 2013 Nucl. Fusion 53073043

[10] Kirschner A et al 2015 J. Nucl. Mater. 463116

[11] Widdowson A et al 2014 Phys. Scr. T 159014010

[12] Brezinsek S 2015 J. Nucl. Mater. 46311

[13] Brezinsek S et al 2014 Nucl. Fusion 54103001

[14] Coad J P et al 2001 J. Nucl. Mater. 290-3 224

[15] von Keudell A et al 1999 Nucl. Fusion 391451

[16] Kreter A et al 2009 J. Nucl. Mater. 39038

[17] Coad J P et al 2014 Phys. Scr. T 159014012

[18] Ivanova D et al 2014 Phys. Scr. T 159014011

[19] Mayer M et al 2015 Phys. Scr. 2016014051 